\title{
ON THE FINITE DETERMINATION OF CRITICAL LATTICES
}

\section{HARVEY COHN ${ }^{1}$}

1. Introduction. We are concerned here with the determination of critical lattices with respect to a given finite convex body $K$ containing the origin and symmetric to it. According to the usual terminology, a critical lattice is a $K$-admissible lattice of minimal determinant, while a $K$-admissible lattice is one all of whose points, except the origin, lie on or outside $K$. It is not hard to show $[5, \text { p. 13 }]^{2}$ that a critical lattice exists for every such $K$.

In $d$-dimensional space a lattice is determined by $d^{2}$ real variables or $d$ vectors $\alpha_{1}, \alpha_{2}, \cdots, \alpha_{d}$. If $K$ is defined by a gauge function, in vector form $\Phi\left(x_{i}\right) \leqq 1$, then the problem is to minimize the determinant of the $\alpha_{1}$ subject to the infinitude of side conditions

$$
\Phi\left(p_{1} \overrightarrow{\alpha_{1}}+p_{2} \overrightarrow{\alpha_{2}}+\cdots+p_{d} \overrightarrow{\alpha_{d}}\right) \geqq 1
$$

where $\left(p_{i}\right)$ runs through all integer sets except the origin. Now, as mentioned earlier it is possible to show the existence of a critical lattice of positive determinant, in fact, by the use of a compactness argument. We shall show, however, by means of a theorem in the companion paper [1], that the infinite set (1) can be reduced to a finite set whose number of inequalities depends only on $d$. Thus, for instance, we are assured that when $K$ is bounded by algebraic surfaces, then among the (possibly continuous) set of critical lattices, a lattice with an algebraic basis can be found.

Results such as these are implied in the classical reduction theory of quadratic forms and of star bodies [2]. Indeed, Minkowski [3, pp. $51,101]$ solved the problem for convex bodies explicitly when $d=2$ and 3, giving the most economical set of inequalities. The sets given here will be far from economical, but the methods will be comparitively simple and geometrically intuitive.

2. Finite bases lemma. Let $\overrightarrow{\omega_{1}}, \overrightarrow{\omega_{2}}, \cdots, \overrightarrow{\omega_{d}}$ be a set of $d$ independent vectors on the surface of the finite symmetric convex body $K$, and belonging to a $K$-admissible lattice. Then the lattice has a basis from the following finite set:

Presented to the Society, November 25, 1950; received by the editors August 30, 1950.

${ }^{1}$ The author wishes to acknowledge the helpful advice of Mr. C. A. Rogers and of a referee.

${ }^{2}$ Numbers in brackets refer to the bibliography at the end of the paper. 


$$
\begin{aligned}
& \overrightarrow{\Omega_{1}}=\frac{b_{11} \omega_{1}}{a_{1}} \\
& \overrightarrow{\Omega_{2}}=\frac{b_{21} \overrightarrow{\omega_{1}}}{a_{1} a_{2}}+\frac{b_{22} \overrightarrow{\omega_{2}}}{a_{2}}, \\
& \overrightarrow{\Omega_{d}}=\frac{b_{d 1} \overrightarrow{\omega_{1}}}{a_{1} a_{2} \cdots a_{d}}+\frac{b_{d 2} \overrightarrow{\omega_{2}}}{a_{2} \cdots a_{d}}+\cdots+\frac{b_{d d} \overrightarrow{\omega_{d}}}{a_{d}},
\end{aligned}
$$

where the $a_{i}$ and $b_{i j}$ are integers satisfying the conditions:

$$
a_{k}>0, \quad b_{k, k}=1, \quad\left|b_{k l} / a_{k} \cdots a_{l}\right| \leqq \frac{1}{2} a_{l}^{-1},
$$

and

$$
a_{1} a_{2} \cdots a_{d} \leqq d !
$$

This result is due to Minkowski [4, p. 189]; we therefore simply note some special aspects of the lemma which tie in with familiar results. In case there are more than $d$ vectors (and their negatives) on the surface, let us choose $\left(\omega_{i}\right)$ so as to produce a minimal positive determinant. Then for $d=2$ the basis can always be chosen as $\left(\omega_{1}, \omega_{2}\right)$ itself, while for $d=3$ it can be chosen as $\left(\omega_{1}, \omega_{2}, \omega_{3}\right)$ or $\left(\omega_{1}, \omega_{2},\left(\omega_{1}+\omega_{2}+\omega_{3}\right) / 2\right)$, as was known to Minkowski [3, p. 101]. If $K$ is specialized to an ellipsoid, then for $d \leqq 3$ only the basis $\left(\omega_{i}\right)$ will occur.

3. Reduction to finiteness. In the search for critical lattices, we first restrict the $K$-admissible lattices to the subclass having $d$ independent vectors on the surface of $K$. This is no restriction since every $K$-admissible lattice can be contracted to a $K$-admissible lattice with the further property. We then let $\left(\omega_{i}\right)$ be such a set of $d$ independent vectors. By the finite bases lemma, the lattice has as its basis a set $\left(\Omega_{i}\right)$ chosen from among the finite collection in (2).

Then the conditions (1), subsidiary to the minimizing of the determinant of the $\Omega_{i}$, can be rewritten as $\Phi\left(\sum p_{i} \Omega^{\rightarrow}\right) \geqq 1$, where $\left(p_{i}\right)$ are still all integral lattice points, not the origin, and where equality is achieved when the argument of $\Phi$ is one of the vectors $\omega_{\text {. }}$ $=\sum_{j=1}^{i} a_{i j} \Omega_{j}$, from (2). Now the convex body $K$ determines a conjugate convex body $K_{0}$ in the $p$-space by means of the inequality in the $p_{i}$ : $\Phi\left(\sum p_{i} \Omega_{i}\right) \leqq 1$. Furthermore $K_{0}$ contains the $2^{d}$-hedron determined by the vectors $\pm\left(a_{11}, 0,0, \cdots, 0\right), \pm\left(a_{21}, a_{22}, 0, \cdots, 0\right), \cdots$, 
$\pm\left(a_{d 1}, a_{d 2}, a_{d 3}, \cdots, a_{d d}\right)$. But these $2^{d}$-hedra are finite in number, one for each basis, and they contain the origin and hence a hypersphere of radius $\rho_{d}$, depending on $d$ alone. To be more specific, using the estimates $a_{11} \cdots a_{d d}<d$ ! and $a_{j j} \geqq\left|a_{k j}\right|$ for $k>j$ (an easy consequence of $(2 \mathrm{a}))$, we find that for instance $\rho_{d} \geqq d^{-1 / 2}(d !)^{-3 / 2}$. Hence by the main theorem of the companion paper [1], $k$ will contain no $p$-integral lattice points, other than the origin, in its interior if it fails to contain those in a hypersphere of radius $C_{d \rho_{d}}^{-(d-1)}$.

Thus the system of inequalities (1) can now be replaced by the finite collection of the finite systems (one system for each basis)

$$
\begin{array}{lr}
\Phi\left(\sum_{i=1}^{k} a_{i k} \overrightarrow{\Omega_{k}}\right)=1, & 1 \leqq k \leqq d, \\
\Phi\left(\sum_{i=1}^{1} p_{i} \overrightarrow{\Omega_{i}}\right) \geqq 1, \text { for } 0<\left(\sum p_{i}^{2}\right)^{1 / 2}<C_{d \rho_{d}}^{-(d-1)} \text { ( } p_{i} \text { integral). }
\end{array}
$$

In principle, of course, we select the basis whose system gives the smallest determinant; and in each system we would have had to change some inequalities to equality and to ignore many more. When $d>3$, however, there seems to be no easy way to determine a minimal system.

\section{BIBLIOGRAPHY}

1. Harvey Cohn, On finiteness conditions for a convex body, Proceedings of the American Mathematical Society vol. 2 (1951) pp. 544-546.

2. K. Mahler, On lattice points in n-dimensional star bodies, Proc. Roy. Soc. London. Ser. A vol. 187 (1946) pp. 151-187.

3. H. Minkowski, Diophantische Approximationen, Leipzig, 1907.

4. - Geometrie der Zahlen, Leipzig, 1910.

5. H. Weyl, C. L. Siègel, and K. Mahler, Geometry of numbers, mimeographed seminar notes, Princeton, 1949.

WAYNE UNIVERSITY 